\title{
Pesquisa Baseada em Design: Um Mapeamento Sistemático da Produção Acadêmica em Acesso Aberto em Língua Portuguesa
}

\author{
Danielle Reis $^{1}$, Tel Amiel ${ }^{1}$ \\ ${ }^{1}$ Faculdade de Educação - Universidade de Brasília (UnB) \\ Campus Darcy Ribeiro - Brasília - DF - Brasil \\ amieleunb.br, prof.daniellereis@gmail.com
}

\begin{abstract}
This work presents a systematic mapping of literature on Design Based Research in education. The basic objective of this study was to map portuguese-language, open acess publications using DBR as an approach. We analyze the adherence of 30 studies to some of the basic principles of DBR. We discuss the implications of our findings, specifically in how articles communicate DBR work, so as to promote the pertinence and challenges of these principles for research in educational technology.

Resumo. Este trabalho apresenta um mapeamento sistemático da literatura sobre Pesquisa Baseada em Design (DBR) em educação. O objetivo principal deste estudo foi o de mapear as publicações em língua portuguesa publicadas em acesso aberto que relatam utilizar a DBR. Analisamos a aderência de 30 estudos a alguns dos princípios básicos de DBR. Discutimos as implicações dos achados para a publicação de artigos que se utilizam da DBR, para que possamos promover a pertinência e desafios de implementação desses princípios para pesquisa em tecnologia e educação.
\end{abstract}

\section{Introdução}

Podemos dividir os objetivos da pesquisa educacional em duas grandes áreas: modificar a prática ou contribuir para o desenvolvimento de conhecimento (Reeves, 2000). No entanto, há uma relação entre ambas as esferas de investigação: espera-se que geração de novos conhecimentos (teorias, metodologias, métodos) possa ter algum impacto nas práticas de ensino. Muito do que se pesquisa hoje no Brasil, tem como prioridade o desenvolvimento de conhecimento, tratando-se de pesquisa de cunho avaliativo ou analítico. São comuns as pesquisas que expõem a condição do ensino, seus problemas e percalços, algumas incluindo recomendações para ação futura. Estudos deste cunho são essenciais para que possamos entender a dinâmica escolar, e identifiquemos problemas a serem estudados e remediados. Idealmente, uma pesquisa avaliativa ou analítica traz à tona questões, problemas, dilemas e fatores, que devem ser posteriormente incorporados em projetos de intervencionistas. Uma pesquisa intervencionista parte do princípio que algum método, técnica, política, ou prática pedagógica poderá transformar os problemas identificados nas pesquisas de análise ou avaliação.

Nessa direção, o modelo de pesquisa conhecido como Pesquisa Baseada em Design (Design-Based Research; DBR) é uma abordagem de investigação com foco no desenvolvimento de intervenções que possam ser realizadas e integradas às práticas 
VIII Congresso Brasileiro de Informática na Educação (CBIE 2019)

Anais do XXX Simpósio Brasileiro de Informática na Educação (SBIE 2019)

educacionais (Matta, Silva e Boaventura, 2014). A DBR apresenta grande potencial gerador de estratégias de interação e colaboração na busca de soluções práticas para os problemas da educação, ao mesmo tempo que visa contribuir para o desenvolvimento de conhecimento teórico-metodológico.

Neste trabalho, apresentaremos um mapeamento sistemático da literatura de DBR apontando seus elementos e recomendações para pesquisa nessa área. O objetivo principal deste estudo é mapear as publicações em língua portuguesa que relatam pesquisas utilizando a DBR e analisar a aderência aos seus princípios básicos. A investigação dos princípios é importante para que o modelo possa ser refinado e para que futuros pesquisadores possam melhor entender seus desafios e potenciais.

\section{O impacto da pesquisa em educação}

A procura por fatores que contribuem para a falta de desenvolvimento educacional no Brasil cai, impreterivelmente nas mãos dos pesquisadores em educação. $O$ investimento em pesquisa tem sido feito em sua grande parte pela esfera pública, cabendo então às instituições públicas a tarefa de pesquisar o ensino público. Cabe ao pesquisador em seu papel social investigar o complexo ambiente escolar no intuito de analisar a educação e identificar práticas educacionais que viabilizem uma melhora no ensino público. No entanto, como já nos indicou há tempos Gatti (2001), o impacto da pesquisa em educação ainda é limitado. Isso pode ser atribuído a alguns fatores, dentre eles a

\footnotetext{
"desvinculação das universidades brasileiras com os níveis básicos de ensino; distanciamento das universidades em relação aos problemas práticos; visão idealizada e teórica da universidade sobre o ensino; falta de contato dos órgãos governamentais com a universidade; o caráter teórico das pesquisas; a inexistência quase total de trabalhos conjuntos;:" (p.77).
}

Seguindo Gatti (e mais recentemente, André, 2006, Kuhlmann, 2014), diversos desses problemas podem ser endereçados por modelos de pesquisa que sejam mais abertos em sua condução e na publicização de seus resultados e que busquem uma articulação mais próxima entre pesquisadores e educadores na identificação e resolução de problemas locais. Nesse sentido, a DBR é um modelo de pesquisa que alia intencionalidades práticas às produções teóricas, devido aos ciclos iterativos de design, implementação, avaliação e redesign, que podem produzir resultados na forma de soluções e/ou produtos gerando novas teorias e princípios para implementação.

Para investigar e entender como a DBR está sendo apropriada em pesquisa educacional, este estudo apresenta a análise de um conjunto de artigos publicados em língua portuguesa que relatam investigações apoiadas na DBR. Mediante um mapeamento sistemático de literatura, fizemos um levantamento de seu uso em pesquisas intervencionistas, analisando a aderência aos princípios fundamentais da DBR.

\section{Pesquisa Baseada em Design}

Concordamos com Nobre et al. (2017) que DBR é "uma tipologia de pesquisa científica, na qual pesquisadores em educação desenvolvem, em colaboração com os participantes, soluções para os desafios/ problemas identificados no contexto escolar." 
VIII Congresso Brasileiro de Informática na Educação (CBIE 2019)

Anais do XXX Simpósio Brasileiro de Informática na Educação (SBIE 2019)

(p. 130). Alguns (McKenney e Reeves, 2014) preferem identificar esse modelo como educational design research (pesquisa em designs educacionais), para enfatizar o enfoque em educação e distinguir o modelo de outras áreas que utilizam o termo design. Aqui, seguimos com a terminologia mais abrangente (DBR).

Amiel e Reeves (2008) indicaram alguns princípios básicos da DBR. O processo se inicia com a identificação conjunta de um problema ou inquietação. Nada impede que pesquisadores a priori proponham um problema de pesquisa. Mas este só pode ser efetivamente definido em negociação com atores locais (professores, gestores, etc.). $\mathrm{Na}$ DBR há sempre uma negociação e contextualização do problema em parceria com atores locais (buscando uma validade ecológica), o que pode ser um processo demorado e cíclico.

DBR preza pelo desenvolvimento de alguma intervenção, no contexto real de sua aplicação contínua e futura. A viabilidade da intervenção de forma parcial ou total em contextos outros pode ajudar a informar a implementação em um contexto final, mas nunca poderá substituí-lo. A intervenção, sendo educacional, pode incluir: um novo software ou hardware educacional, uma nova sequência didática, ou ainda uma nova proposta, processo ou metodologia de ensino. Mesmo incluindo um software ou um hardware, estes nunca são considerados uma intervenção em si. A ferramenta ou dispositivo deve ser compreendido como parte de toda uma contextualização para investigação, um design que envolve atores, contextos, contingências e métodos, que são, em conjunto, o objeto real de estudo (Amiel \& Reeves, 2008).

A investigação deve proceder de forma a ser realizada em iterações, visando o refinamento da intervenção (produto, processo, etc.) bem como a construção de novos conhecimentos teóricos sobre as possibilidades de implementação. DBR reconhece que intervenções em contextos educacionais são processos complexos e sistêmicos, e que raramente uma única intervenção de curto prazo resulta em resultados satisfatórios. Infelizmente, pelos modelos praticados na pesquisa acadêmica (por exemplo, um mestrado de curta duração) estudos de curto prazo e com uma única tentativa de implementação são a tônica em tecnologia e educação. No âmbito prático, o desenvolvimento de soluções, particularmente àquelas envolvendo tecnologia educacional demandam diversos ciclos de refinamento. Em termos teóricometodológicos, a derivação de princípios depende de investigações que permitam um refino contínuo e um olhar para diversos aspectos da intervenção, o que raramente pode ser feito em curto prazo. Na medida em que ciclos são realizados, o entendimento sobre o fenômeno e seu espaço de aplicação aprimoram o entendimento sobre sua aplicabilidade e adequação em diferentes contextos.

\section{O Processo do Mapeamento Sistemático}

O mapeamento sistemático é um tipo de pesquisa muito utilizada quando há um cenário abrangente e que tem por objetivo reunir o máximo de informações disponíveis sobre uma determinada área do conhecimento. Este mapeamento foi conduzido baseado no processo descrito por Ramires et al. (2013), as etapas consistem em: (i) Planejamento - definição das Questões de Pesquisa e elaboração do Protocolo, contendo, dentre outros itens, a definição de fontes de busca, do método de busca do material, e dos critérios de inclusão/exclusão; (ii) Execução - busca de estudos primários, pré-seleção de estudos aplicando os critérios de inclusão e exclusão; e (iii) Análise - síntese das 
VIII Congresso Brasileiro de Informática na Educação (CBIE 2019)

Anais do XXX Simpósio Brasileiro de Informática na Educação (SBIE 2019)

informações. Nas subseções 4.1 e 4.2, são abordadas, respectivamente, as Questões de Pesquisa e o Protocolo da etapa de Planejamento. As etapas de Execução e Análise estão contempladas nas seções 5 e 6 .

\subsection{Questões de Pesquisa}

As questões de pesquisa que norteiam o estudo foram derivadas a partir dos objetivos do mapeamento: QP: Qual panorama da produção acadêmica em língua portuguesa, em acesso aberto, que indicam fazer uso de DBR? $\mathbf{Q P}_{2}$ : As pesquisam que relatam investigação utilizando a DBR aderem aos princípios fundamentais do modelo? $\mathbf{Q P}_{2.1}$ : A análise e definição de problemas práticos foram negociados entre pesquisadores e atores locais? $\mathbf{Q P}_{2.2}$ : Houve desenvolvimento colaborativo de artefatos e/ou métodos informados por teorias, designs e técnicas existentes? $\mathbf{Q P}_{2.3}$ : Os trabalhos produziram reflexões para derivar princípios de melhoria ao design e contribuições teóricometodológicas? $\mathbf{Q P}_{2.4}$ : Foram realizados ciclos iterativos de implementação e investigação em contextos reais de forma colaborativa?

\subsection{Protocolo do Planejamento}

Buscamos artigos que foram publicados em acesso aberto no idioma português. Para isso foram utilizados os seguintes termos de busca de forma individual no texto completo: "Design based research" ou "Development research" ou "DBR" ou "Pesquisa baseada em design" ou "Pesquisa de desenvolvimento". A escolha destes termos foi em razão da tradução e dos possíveis sinônimos da expressão original, evidenciada também em textos previamente investigados. Compilamos os dados em uma tabela e investigamos a pertinência de cada termo. Na investigação dos artigos resultantes de busca de cada termo, encontramos muitos trabalhos que estavam fora de escopo. A sigla DBR, por exemplo, surgiu em vários artigos com uma diversidade de significados incompatíveis com nosso interesse. Decidimos restringir a pesquisa pelo termo original no idioma inglês "Design based research" e "Pesquisa baseada em design".

A pesquisa teve como enfoque a seleção de trabalhos depositados nos repositórios abertos e periódicos: Directory of Open Access Journals (DOAJ), nos Repositórios Científicos de Acesso Aberto de Portugal (RCAAP) na Red de Revistas Científicas de América Latina y el Caribe, España y Portugal (REDALYC), Scielo, @Educa, além da base de publicações indexadas pela Comissão Especial de Informática na Educação (CEIE), que inclui: Revista Brasileira de Informática na Educação (RBIE), Simpósio Brasileiro de Informática na Educação (SBIE), Anais do Workshop de Informática na Escola (WIE), Anais dos Workshops do Congresso Brasileiro de Informática na Educação (WCBIE). Essa escolha justifica-se pelo princípio ético de incluir somente bases e publicações de acesso aberto.

O processo de seleção das publicações foi composto por duas etapas de triagem. Os resultados nos apontaram um total de 90 trabalhos, conforme tabela a seguir.

Tabela 1. Quantidade de publicações nas bases e repositórios abertos. 
VIII Congresso Brasileiro de Informática na Educação (CBIE 2019)

Anais do XXX Simpósio Brasileiro de Informática na Educação (SBIE 2019)

\begin{tabular}{|c|c|c|c|}
\hline $\begin{array}{l}\text { Termos de busca } \\
\text { Repositórios Abertos }\end{array}$ & $\begin{array}{c}\text { "Design } \\
\text { Based } \\
\text { Research" }\end{array}$ & $\begin{array}{c}\text { "Pesquisa } \\
\text { Baseada } \\
\text { em Design" }\end{array}$ & Total \\
\hline DOAJ & 11 & 9 & 20 \\
\hline RCAAP & 21 & 4 & 25 \\
\hline REDALYC & 24 & 12 & 36 \\
\hline Scielo & 1 & 3 & 4 \\
\hline @Educa & 1 & 3 & 4 \\
\hline RBIE & 0 & 0 & 0 \\
\hline SBIE & 0 & 0 & 0 \\
\hline WIE & 0 & 1 & 1 \\
\hline WCBIE & 0 & 0 & 0 \\
\hline Total & 58 & 32 & 90 \\
\hline
\end{tabular}

Dos 90 artigos, 34 foram descartados em uma primeira leitura por não se relacionar a DBR. Para os 56 restantes, foi realizada a leitura do título e resumo para selecionar os artigos relacionados à utilização da abordagem DBR. Aplicamos os seguintes critérios de inclusão: a) trabalhos publicados em qualquer período, sem restrição de data; b) artigos em língua portuguesa; c) o texto deveria tratar sobre DBR no sentido de uma abordagem e d) deveria relatar um estudo empírico. Os critérios de exclusão foram: a) artigos descritivos, com foco em discutir o significado de DBR ou somente problematizar a metodologia, sem relato de investigação; b) artigos em língua estrangeira; c) trabalhos duplicados. Os resumos foram lidos pelos dois pesquisadores, autores deste trabalho, em caso de discordância ou dúvida o texto completo foi lido para decisão de inclusão/exclusão. Na segunda triagem, fizemos a leitura completa dos trabalhos selecionados na primeira triagem, sendo submetidos aos mesmos critérios de inclusão/exclusão. Os trabalhos não foram filtrados por critérios de qualidade. Um total de 30 trabalhos foram considerados para esse mapeamento.

Após o processo de seleção dos trabalhos relevantes ao contexto do estudo, foram registrados os artigos na base do software de gerenciamento e elaborados fichamentos com as informações que seriam analisadas. No espírito do compartilhamento aberto de dados científicos, utilizamos um grupo aberto na plataforma Zotero (https://www.zotero.org/groups/2298096/ dbr_educao_infantil_e_abertura/), um software livre para gerenciar dados bibliográficos e materiais relacionados à pesquisa, um software colaborativo e de código aberto. Isto permitiu organizar e publicar de forma aberta o conjunto de trabalhos contendo além dos metadados, o link para o texto completo bem como a resenha feita para cada um, buscando total transparência do processo de mapeamento sistemático.

\section{Resultados}


VIII Congresso Brasileiro de Informática na Educação (CBIE 2019)

Anais do XXX Simpósio Brasileiro de Informática na Educação (SBIE 2019)

Seguindo o Protocolo de Planejamento, após os critérios de inclusão/exclusão, foram selecionados o total de 30 artigos em língua portuguesa que relatam investigações utilizando a DBR, o que responde a questão de pesquisa $\mathbf{Q} \mathbf{P}_{1}$. Para esse mapeamento, com base nos princípios fundamentais selecionados para DBR, buscamos identificar nos trabalhos analisados se os mesmos se adequavam aos princípios básicos da DBR, com isso visamos melhor entender de que maneira DBR está sendo transposta em estudos empíricos, enfoque principal desse trabalho.

Os princípios foram sistematizados da seguinte forma: 1. o problema foi negociado pelos pesquisadores e participantes em colaboração; 2 . desenvolveu algum produto e/ou processo; 3 . houve intervenção, ou seja, se foi levado para campo; 4. buscou apresentar contribuição teórico-metodológica; 5. apresentou contribuição prática e, por fim, 6. a quantidade de ciclos iterativos realizados. Os dados da Tabela 1 indicam a quantidade de artigos que efetivou (Sim) ou não efetivou (Não) cada princípio, conforme explanado anteriormente.

Tabela 2. Princípios da DBR nos artigos analisados.

\begin{tabular}{|l|l|l|}
\hline \multicolumn{1}{|c|}{ Princípios } & \multicolumn{1}{c|}{ Sim } & \multicolumn{1}{c|}{ Não } \\
\hline 1. Problema negociado & 8 & 22 \\
\hline 2. Desenvolveu produto/processo & 30 & 0 \\
\hline 3. Levou para campo & 28 & 2 \\
\hline 4. Contribuição teórica & 1 & 29 \\
\hline 5. Contribuição prática & 29 & 1 \\
\hline
\end{tabular}

Em resposta à questão de pesquisa $\mathbf{Q P}_{2}$, observamos que dos 30 artigos selecionados, em 8 pesquisas o problema foi negociado em colaboração com os participantes e em 22 o problema foi elaborado previamente pelos investigadores. Todos os trabalhos desenvolveram algum produto ou processo, sendo que 28 deles foram levados para campo. Apenas 1 dos trabalhos resultou em uma discussão visando contribuição teórico-metodológica e 29 apresentaram efetiva retroalimentação, para contribuição ao design ou prática.

Na Tabela 2 apresentamos a quantidade de iterações realizadas nos estudos como resposta da questão de pesquisa $\mathbf{Q} \mathbf{P}_{2.4}$. Sinalizamos 0 para os trabalhos que não efetuaram ciclos iterativos; 1 para os que realizaram a implementação (uma vez) e 2 para os que realizaram uma iteração (implementação + iteração única).

Dentre os artigos selecionados, 2 estudos não foram a campo, 25 efetuaram a implementação somente e 3 fizeram uma implementação seguido de uma iteração.

\section{Análise}

Esta seção mostra o mapeamento da aderência a alguns dos princípios básicos de DBR nos trabalhos analisados, como respostas aos questionamentos levantados nas questões de pesquisas.

$\mathbf{Q P}_{2.1}$ “A análise e definição de problemas práticos foram negociados entre pesquisadores e atores locais?". Uma das fortes recomendações da DBR é que o problema seja definido de forma compartilhada com os participantes. Porém os dados indicam uma lacuna neste princípio, visto que esta característica não está presente na maioria das pesquisas analisadas. Uma quantidade significativa de trabalhos não 
VIII Congresso Brasileiro de Informática na Educação (CBIE 2019)

Anais do XXX Simpósio Brasileiro de Informática na Educação (SBIE 2019)

descreveu um processo de negociação na definição de problemas de pesquisa. De maneira a refinar um problema, Bittencourt e Struchiner (2015), "A articulação da temática da doação de sangue e o ensino de biologia no Ensino Médio: uma pesquisa baseada em design", os autores relatam que esta fase foi realizada por meio de entrevistas com os professores com o intuito de identificar suas abordagens sobre questões relativas às temáticas sociais, à doação de sangue e às TICs em sala de aula. Para além, no trabalho de Struchiner e Giannella (2016) "Com-Viver, Com-Ciência e Cidadania: uma Pesquisa Baseada em Design integrando a Temática da Saúde e o Uso de Tecnologias Digitais de Informação e Comunicação na Escola”, foram realizados centros de estudos dedicados à identificação do problema educativo. De acordo com as autoras, estes encontros produziram uma série de reflexões e discussões sobre a visão de saúde dos professores e a possibilidade de integração desta temática em suas disciplinas.

$\mathbf{Q P}_{2.2}$ "Houve desenvolvimento colaborativo de artefatos elou métodos informados por teorias, designs e técnicas existentes?" Ao analisar os 30 trabalhos selecionados, observamos que todas as pesquisas desenvolveram alguma aplicação e produziram produtos educacionais ou processos pedagógicos. Por exemplo, na pesquisa "Implementação de mídias em dispositivos móveis: um framework de aplicação em grande escala na educação a distância" (Mülbert e Pereira, 2017) desenvolveu-se um livro didático eletrônico adaptado para uso em dispositivos móveis. A partir dessa iniciativa extraiu-se o conhecimento alcançado para propor um framework de apoio a implementação de iniciativas similares. Batista e Siqueira (2017) no artigo "A inserção da Física Moderna e Contemporânea em ambientes reais de sala de aula: uma sequência de ensino-aprendizagem sobre a radioatividade" formularam uma sequência de ensino-aprendizagem (TLS) sobre o tópico radioatividade a partir do modelo de estrutura didática e desenvolvida nos pressupostos da abordagem problematizadora. A TLS foi implementada em escolas públicas e, segundo os autores, produziu conhecimentos relevantes a serem compartilhados com a comunidade do ensino de Física.

$\mathbf{Q P}_{2.3}$ "Os trabalhos produziram reflexões para derivar princípios de melhoria ao design e contribuições teórico-metodológicas?" Quanto à característica intervencionista da DBR, os resultados apontam que na maioria dos artigos analisados ocorreu intervenção prática. No entanto, é possível observar lacunas em relação à elaboração teórica, o foco dos estudos está no trabalho empírico. O estudo de Santos e Rossini (2015), "Design-interativo aberto: um dispositivo da pesquisa-formação na cibercultura", evidenciou contribuição teórico-metodológico com o desenvolvimento de atos de currículo inovadores para a atualização da metodologia pesquisa-formação. Os atos de currículo foram criados como suporte para situações de ensino e aprendizagem que propiciem a produção de materiais didáticos digitais. Dentre os trabalhos que resultaram em soluções práticas, a pesquisa "Recursos Educacionais Abertos: Acesso Gratuito ao Conhecimento?” (Mazzardo, Nobre e Mallmann, 2017) elaborou um curso de formação de professores para o uso e adaptação de recursos educacionais abertos (REA), com o propósito de possibilitar o conhecimento e a integração de REA nos materiais e nas atividades didáticas dos participantes.

$\mathbf{Q P}_{2.4}$ "Foram realizados ciclos iterativos de implementação e investigação em contextos reais de forma colaborativa?" Reeves (2000), destaca a necessidade de realizar investigação rigorosa e reflexiva para testar e refinar ambientes de 
VIII Congresso Brasileiro de Informática na Educação (CBIE 2019)

Anais do XXX Simpósio Brasileiro de Informática na Educação (SBIE 2019)

aprendizagem inovadores, bem como para estabelecer novos princípios de design, o que define a abordagem iterativa da DBR. Com base nas publicações selecionadas, notamos grande lacuna quanto à iteração nas pesquisas, consideramos que um único ciclo, como foi realizado em grande parte dos trabalhos, dificilmente é suficiente para o refinamento da solução prática aplicada, sendo necessários dois ou mais ciclos iterativos. Isso é evidente no trabalho de Pessanha e Pietrocola (2016) "O ensino de estrutura da matéria e aceleradores de particulas: uma pesquisa baseada em design", que elaborou um curso de curta duração sobre o tópico estrutura da matéria e aceleradores de partículas. Os autores relatam que uma primeira versão do curso foi desenhada, esta versão foi implementada com um grupo de alunos, e após, com base nos resultados de aprendizagem e outros aspectos didáticos observados durante a implementação, seguiu-se um processo de redesenhos e novas implementações. Do mesmo modo ocorreu no estudo de Dias e Reis (2017) "O Desenvolvimento de Atividades Investigativas com Recurso à Web 2.0 no Âmbito da Investigação e Inovação Responsáveis", onde foram criados módulos de aprendizagem com diferentes estratégias didáticas para a educação científica no ensino básico. Conforme os autores relatam, os módulos foram implementados em contexto de sala de aula. Os alunos e as professoras avaliaram os módulos de ensino e deram algumas sugestões, a partir das quais foram efetuadas as devidas alterações e realizada nova implementação.

A investigação apresentada aqui foi feita somente com base nas informações apresentadas nos artigos. Mencionamos isso, porque é prática comum relatar em trabalhos os resultados parciais ou preliminares de uma pesquisa. Nesse caso, poderíamos imaginar que alguns trabalhos estão relatando tão somente um ciclo inicial de implementação, na promessa de que outras iterações estão por vir. No entanto, visando o alinhamento metodológico, nos parece importante que, na contramão de relatos de teste com usuários, ou investigações de curto prazo, as investigações alinhadas à DBR devem primar por divulgar resultados somente quando ao menos uma iteração (dois ciclos) fosse concluída. Dado que a iteração é tão essencial ao processo, nos parece importante que trabalhos nessa área discutam as dificuldades e nuances de desenvolver, aplicar e gerar conhecimento através de ciclos iterativos de implementação.

O mesmo pode ser dito para a produção de conhecimento teórico-metodológico e prático. A melhoria de processos e produtos é inerente à DBR. Por isso é necessária a reflexão acerca de questões teórico- metodológicas. Trata-se de um olhar para a transparência e integridade dos dados e da análise, que permitam identificar fatores e elementos importantes para o sucesso de uma implementação. Diferentemente de outros modelos de pesquisa, não se trata de buscar uma intervenção estéril que possa funcionar in toto e sem adaptação à qualquer contexto. Os fatores ou princípios, descritos de maneira detalhada, podem ajudar outros pesquisadores e levar os conhecimentos e os produtos desenvolvidos para outros contextos com suas devidas adaptações. Trata-se de descrever e estudar os elementos do contexto que podem contribuir para o sucesso ou características específicas da intervenção. Esse conhecimento heurístico pode ajudar a entender que a intervenção $\mathrm{X}$, pode funcionar melhor no contexto $\mathrm{Y}$, se estiver atenta a fatores como Z, por exemplo (Fishman et al., 2013; McKenney e Reeves,2014).

\section{Conclusões}


Os trabalhos apresentados foram investigados somente quanto a sua aderência a alguns dos princípios básicos do modelo, a partir da busca por respostas para as questões de pesquisa. Com isso, pretendemos compreender de que maneira pesquisadores estão se apropriando dos elementos que fazem com que DBR seja peculiar, e ao mesmo tempo complexa em sua adoção.

A análise dos textos nos indica alguns padrões. Porém não se trata de tecer críticas ao teor dos estudos e trabalhos realizados, a qualidade das intervenções ou produtos desenvolvidos. Sabemos das dificuldades de realizar pesquisa aplicada, em campo, em contextos educacionais. Sabemos também das pressões que existem para contínua publicação de trabalhos acadêmicos, o que pode levar a publicação fragmentada de estudos (que potencialmente teriam novos ciclos e iterações). É importante, no futuro, aumentar o escopo da busca para incluir publicações em outros idiomas e outros repositórios, além de realizar análises mais detalhadas de relações entre autores e temas, etapas de ensino que foram realizadas as pesquisas, tipo de ensino - formal ou informal -, dentre outros, informações que podem fomentar redes e conexões.

Vemos esse trabalho como uma forma de analisar e trazer contribuições para uma discussão sobre como podemos fomentar pesquisa na área de tecnologia e educação voltada para os princípios delineados acima. Ao identificar algumas possíveis lacunas vemos oportunidades de engajamento e discussão acerca das dificuldades e nuances de sua implementação na prática de pesquisa. Os princípios abarcados por DBR não são consenso absoluto, mas a um nível geral de concordância quanto aos seus elementos centrais (McKenney e Reeves, 2014). Estes são ao mesmo tempo um alerta sobre lacunas em nossas práticas de pesquisa atuais, bem como um norte para como podemos conduzir nossas investigações. Sem um novo olhar para como publicamos nossos trabalhos e posterior discussão acadêmica sobre seus desafios e possibilidades desses princípios, dificilmente avançaremos na percepção de qualidade e aplicabilidade das nossas pesquisas.

\section{Bibliografia}

AMIEL, T.; REEVES, T. C. Design-Based Research and Educational Technology: Rethinking Technology and the Research Agenda. Journal of Educational Technology and Society, v. 11, n. 4, p. 29-40, 2008.

ANDRÉ, M. A jovem pesquisa educacional brasileira. Diálogo Educacional, v. 6, n. 19, p. 11-24, 2006.

BATISTA, C. A. DOS S.; SIQUEIRA, M. A inserção da Física Moderna e Contemporânea em ambientes reais de sala de aula: uma sequência de ensinoaprendizagem sobre a radioatividade. Caderno Brasileiro de Ensino de Física, v. 34, p. 880-902, dez 2017.

DIAS, C. P.; REIS, P. O Desenvolvimento de Atividades Investigativas com Recurso à Web 2.0 no Âmbito da Investigação e Inovação Responsáveis. Sisyphus Journal Of Education, v. 5, n. 3, p. 68-84, 2017.

FISHMAN, B. et al. Design-based implementation research: An emerging model for transforming the relationship of research and practice. Design-based implementation research: Theories, methods, and exemplars. National Society for the Study of Education Yearbook, v. 112, n. 2, p. 136-156, 2013. 
GATTI, B. A. Implicações e perspectivas da pesquisa educacional no Brasil contemporâneo. Cadernos de Pesquisa, v. 113, n. Julho, p. 65-81, 2001.

KUHLMANN JR, M. Produtivismo acadêmico, publicação em periódicos e qualidade das pesquisas. Cadernos de Pesquisa, v. 45, n. 158, p. 838-855, 11 dez. 2015.

LEYLANE PORTO BITTENCOURT; MIRIAM STRUCHINER. A articulação da temática da doação de sangue e o ensino de biologia no Ensino Médio: uma pesquisa baseada em design. Ciência e Educação (Bauru), v. 21, n. 1, mar. 2015.

MATTA, A. E. R.; SILVA, F. DE P. S. DA; BOAVENTURA, E. M. Design-based research ou pesquisa de desenvolvimento: Metodologia para pesquisa aplicada de inovação em educação do século XXI. Revista da FAEEBA - Educação e Contemporaneidade, v. 23, n. 42, p. 23-36, 2014.

MAZZARDO, M. D.; NOBRE, A. M. F.; MALLMANN, E. M. Recursos Educacionais Abertos: Acesso Gratuito ao Conhecimento? EaD em Foco, v. 7, n. 1, p. 27-36, 2017.

MCKENNEY, S.; REEVES, T. C. Educational design research. In: Handbook of research on educational communications and technology. [s.l.] Springer, 2014. p. 131-140.

MIRIAM STRUCHINER; TAÍS RABETTI GIANNELLA. Com-Viver, Com-Ciência e Cidadania: uma Pesquisa Baseada em Design integrando a Temática da Saúde e o Uso de Tecnologias Digitais de Informação e Comunicação na Escola. ComViver, Com-Ciência e Cidadania: uma Pesquisa Baseada em Design integrando a Temática da Saúde e o Uso de Tecnologias Digitais de Informação e Comunicação na Escola, v. 14, n. 3, p. 942 - 969, setembro 2016.

MÜLBERT, A. L.; PEREIRA, A. T. C. Implementação de mídias em dispositivos móveis: um framework de aplicação em grande escala na educação a distância. RIED. Revista Iboeroamericana de Educación a Distancia, v. 20, n. 2, p. 233-254, 2017.

NOBRE, A. M. F. et al. Princípios teórico-metodológicos do design-based research (DBR) na pesquisa educacional tematizada por recursos educacionais abertos (REA). Revista San Gregorio, v. 16, n. Edição especial, p. 128-141, 2017.

PESSANHA, M.; PIETROCOLA, M. O ensino de estrutura da matéria e aceleradores de partículas: uma pesquisa baseada em design. Revista Brasileira de Pesquisa em Educação em Ciências, v. 16, n. 2, p. 361-388, 2016.

RAMIRES, L. O. et al. Como a Tecnologia Assistiva tem auxiliado o Processo de Ensino/Aprendizagem? Mapeamento Sistemático a partir dos Trabalhos Publicados no SBIE. In: XXIV Simpósio Brasileiro de Informática na Educação - SBIE. p. 447-456, 2013. Disponível em: <https://www.brie.org/pub/index.php/sbie/article/view/2523/2181>.

REEVES, T. C. Socially responsible educational technology research. Educational Technology, v. 40, n. 6, p. 19-28, 2000.

SANTOS, E. O. DOS; ROSSINI, T. S. S. Design-interativo aberto: um dispositivo da pesquisa-formação na cibercultura. Revista e-Curriculum, v. 13, n. 3, p. 569588, jul. 2015. 